\title{
Retos y oportunidades del SEAE sobre el terreno
}

\author{
Carmelo Angulo Barturen \\ Diplomático \\ Embajador en misión especial para la Cumbre Mundial de Microcrédito
}

Sumario: I. Introducción. - II. Los retos frente al despliegue exterior de la UE. - III. Los retos al interior de los servicios exteriores. - IV. Conclusión.

Resumen: A partir de su amplia experiencia diplomática, el autor ofrece algunas reflexiones sobre las implicaciones de la puesta en acción del Servicio Europeo de Acción Exterior (SEAE) para la proyección de la Unión Europea en el mundo. Tras algunas observaciones de carácter general el texto se centra sucesivamente en dos aspectos: la proyección exterior del SEAE en el medio diplomático internacional, su impacto al interior de los servicios exteriores de los Estados miembros, y sus implicaciones específicas para el caso español.

Palabras clave: UE, SEAE, diplomacia, servicio exterior

Abstract: In this brief contribution, the author, an experienced diplomat, offers his views about the implication of the new European External Action Service for the EU's international role. After some general considerations it addresses consecutively two different aspects: first, the problems related to the EEAS's global deployment in the diplomatic milieu; and second, its implications for existing national foreign services and more specifically for the Spanish case.

Keywords: EU, EEAS, diplomacy, foreign service

\section{Introducción}

La crisis financiera internacional y sus repercusiones sobre Europa y el euro no han sido las mejores circunstancias para el nacimiento del Servicio Europeo de Acción Exterior (SEAE). La compleja encrucijada en la que se encuentra la UE y sus profundas contradicciones, exacerbadas por la falta de políticas fiscales y económicas compartidas, han creado un profundo desánimo ciudadano, desvalorizado el papel de Europa en el mundo y han puesto en sordina, por no decir, en un segundo y lejano plano, el nacimiento del SEAE sobre el que se venía trabajando con sigilo pero con destreza en la última década y que viene a convertirse en la herramienta de apoyo esen- 
cial para el trabajo de la nueva Alta Representante ${ }^{1}$. Estas contradicciones, como subraya Felipe González, han afectado a la acción exterior de la UE ya que «mientras los ciudadanos (europeos) creen en la eficacia de hablar con una sola voz en las distintas instancias y foros internacionales, en porcentajes que llegan casi al $80 \%$, los responsables políticos argumentan al contrario: que sus opiniones públicas no estarían dispuestas a ceder (estas) parcelas de soberanía nacional» ${ }^{2}$.

Sin embargo el tema no deja de ser trascendente para los europeos. Por primera vez, se trata de poner en marcha un servicio diplomático común, instaurado por el Tratado de Lisboa, que trate de dar sustento y acompañamiento eficaz a una política exterior común que nos dé un auténtico protagonismo en la escena mundial. Desde cualquier posición, y por supuesto desde España, es una excelente noticia que celebramos aunque la figura de la Alta Representante de Asuntos Exteriores y Política de Seguridad en sus primeros meses de andadura haya estado sometida a cuestionamientos. Estamos hablando, si las restricciones presupuestarias lo permiten, del potencial derivado de tener cerca de 140 Delegaciones (Embajadas) en el mundo, conducidas por más de 1600 funcionarios muy cualificados, que se multiplicarán en los próximos años, procedentes de la Comisión, el Consejo y la diplomacia de los 27 países miembros y que serán comandados desde Bruselas por una potente estructura ordenada por áreas geográficas y temáticas al modo de un auténtico ministerio de acción exterior ${ }^{3}$.

Es lógico que los primeros anuncios, gestos, nombramientos y reglamentos de aplicación, den pábulo a una cierta desorientación sobre todo porque ocurre en un momento de escasa presencia europea en la actual coyuntura mundial y porque las propias instituciones europeas (Consejo, Comisión y Parlamento) y los países miembros intentan presionar e influir en la nueva estructura que tardará algunos años en tener vuelo y singularidad propia $^{4}$.

Llegar hasta este estadio ha sido enormemente complicado y fruto de una sutil negociación con visiones e intereses a menudo contrapuestos. Por una parte, como señala Francisco Aldecoa, aunque los principios de la po-

${ }^{1}$ Una panorámica general de excepcional interés sobre esos grandes desafíos nos la ofrece: EMERSON, M. et al., Upgrading the EU's Role as Global Actor: Institutions, Law and the Restructuring of European Diplomacy, CEPS/Egmont Institute, 2011. Disponible en: http://www.egmontinstitute.be/news/11/110125-Book-Uprading-EU-as-GlobalActor.html

2 Vid. GONZÁLEZ, F. «Europa en la encrucijada» en El País, 10 de enero de 2011.

3 Vid. organigrama del SEAE en: http://eeas.europa.eu/background/docs/eeas_prov_ organisation_en.pdf

${ }^{4}$ Como muestra de las inquietudes que despierta la creación del SEAE vid.: GRÄSSLE, I., «Analyse critique: La création du Service européen d'action extérieure» en Questions d'Europe, n. 194, 2011. Disponible en http://www.robert-schuman.eu/archives_questions_europe.php 
lítica exterior europea - democracia y derechos humanos, libre comercio, multilateralismo y regionalización -, se enuncian en el Consejo Europeo de Lisboa de 1992 y se recogen en el Tratado de Maastricht, no se consolidan hasta casi un decenio después en el proyecto de Constitución Europea y el Tratado de Lisboa ${ }^{5}$. Este, en su artículo 3.5 y particularmente en el 21, establece con claridad los principios que inspirarán la acción de la Unión en la escena internacional en una línea de responsabilidad que promueve valores, establece alianzas y propicia «soluciones multilaterales a los problemas comunes, en particular en el marco de las Naciones Unidas» ${ }^{6}$. Lo mismo se podría decir del complicado proceso para crear una política de cooperación al desarrollo compartida que nos ha llevado a convertirnos en los primeros donantes del planeta, con prácticamente un $60 \%$ del total de la AOD, y que es fruto, como subraya Francesc Granell, de «la progresiva ampliación de la agenda para el desarrollo y la evolución que han experimentado las doctrinas mundiales sobre la eficacia de la cooperación.... ${ }^{7}$. No en vano, uno de los aspectos más controvertidos durante la negociación del SEAE fue precisamente el modo en que su puesta en acción podía afectar a la política de cooperación al desarrollo y a la acción humanitaria.

Pero a pesar de los sólidos textos legales y las declaraciones pomposas, los precedentes y evidencias concretas no son demasiado halagüeñas. Por una parte, los avances en las definiciones se han visto contrastados por las paradojas de los intereses que con frecuencia han puesto a la economía y la seguridad por encima de la solidaridad y la institucionalidad democrática en una suerte de hipocresía que tiene su sustento en la propia Declaración sobre la Globalización de 2007 en donde queda claro la importancia de los intereses comerciales y empresariales como describe con precisión Sotillo ${ }^{8}$. Por otra, ha sido muy difícil entender y gestionar ya en el terreno operativo, en los países y momentos en los que se daba, los dobles y triples sombreros que sobre el terreno ejercían los Representantes de la Comisión, luego Embajadores, los del Consejo y, cuando existían, los Enviados especiales.

Para los que nos ha tocado, desde nuestra incorporación a Europa y desde diferentes posiciones, trabajar codo a codo en el terreno sirviendo como Embajadores o diplomáticos a España o, en mi caso, a las NN.UU e

5 Vid. ALDECOA, F., «El fundamento de la dimensión exterior del modelo europeo: democracia y derechos humanos» en Revista Española de Desarrollo y Cooperación. vol. 18, 2006, p. 26 y ss.

${ }^{6}$ Ibid.

7 Vid. GRANELL, F., «La política de cooperación al desarrollo de la unión Europea» en Información Comercial Española, n. 831, 2006, p.139.

8 Ver SOTILLO, J.A., «La Unión Europea ante la democracia y los derechos humanos: entre los principios de la superpotencia civil y la defensa de los intereses» en Revista Española de Desarrollo y Cooperación, n. 23, 2009, pp. 67-84. 
incluso gestionar algunas presidencias semestrales, la experiencia ha sido compleja y, a menudo, bastante decepcionante en varios aspectos:

- Frente a un perfil claramente profesional o de confianza de los Embajadores de los países miembros, aparecía una figura, generalmente mucho más técnica, que representaba de manera ambivalente a una solo instancia comunitaria pero que cubría sin embargo no solo las capacidades de RELEX sino los recursos cuantiosos de la cooperación y las otras competencias comunitarias (agricultura, pesca etc.) y que corporizaba la delegación del Parlamento y la ciudadanía europea. Como resultado de la creación del SEAE esas dos culturas profesionales ahora se encontrarán no una frente a otra, sino juntas, en el seno de una misma representación, lo que sin duda constituye una innovación de gran importancia.

- Esta extraña sensación, superada a veces por la empatía personal, quedaba además subsumida por la heterogeneidad del grupo de Embajadas europeas, cada una de tamaño y nivel muy variado y con posiciones políticas e intereses bilaterales dispares y, a veces, en claro antagonismo y competencia. La creación del SEAE constituye sin duda una gran oportunidad para repensar la necesaria articulación de esa heterogeneidad dentro de un esquema que por su propia naturaleza parece exigir un renovado esfuerzo de coordinación. A su vez, la atención explícita al debido equilibrio geográfico y de género en los nombramientos viene a subrayar el carácter innovador del SEAE en el propio medio diplomático.

- Las Embajadas de la Comisión gestionaban a menudo programas y presupuestos casi desconocidos para las embajadas de los países miembros. Los niveles de coordinación o articulación entre Comisión y Embajadas en los temas educativos, culturales, seguridad, DD.HH etc., han sido mínimos o poco efectivos, más allá de algunas presentaciones generales en las reuniones de Jefes de Misión o de los consejeros sectoriales. En los temas económicos y comerciales se competía duramente y en los consulares las divergencias operativas y el sistema de visados, mejorado tras Schengen, estaban sometidos a interpretaciones diferenciadas 9 . A menudo los viajes y programas de los Comisarios, delegaciones de alto nivel o de parlamentarios, eran conocidos muy a última hora y muchos rehuían el contacto con los Embajadores o lo hacían de manera muy protocolar y superficial.

9 Sobre las transformaciones de la función consular en la Unión Europea, vid: FERNÁNDEZ, A.M., «Europeanisation of Consular Affairs: The Case of Visa Policy» en Clingendael Discussion Papers in Diplomacy, n. ${ }^{\circ}$ 105, 2006. Disponible en http://www.nbiz.nl/publications/ 2006/20061000_cdsp_paper_dip.pdf 
- La articulación con el Sistema de NN.UU y las instituciones financieras internacionales ha sido hasta ahora escasa y frecuentemente competitiva, perdiéndose en los países de cooperación intensiva la oportunidad de coordinar más eficientemente esfuerzos en las ayudas humanitarias, los esquemas de reducción de deuda o la programación para alcanzar los $\mathrm{ODM}^{10}$. Con esos precedentes no puede sorprender el reciente fracaso cosechado por la UE en la Asamblea General de Naciones Unidas, en septiembre de 2010, cuando la propuesta presentada por la Presidencia del Consejo para renovar y mejorar los mecanismos actuales de representación y observación de la UE en el seno del sistema de NN.UU ${ }^{11}$. Sin embargo la coincidencia de la crisis financiara global con la creación del SEAE podría llevar a plantear de manera más audaz la necesidad de repensar la representación de la UE en organismos multilaterales que no pueden demorar una revisión interna como el propio $\mathrm{FMI}^{12}$.

- En los países en los que uno o varios países miembros tenían posiciones de liderazgo o de presencia masiva, como nos ocurre a menudo en América Latina, la pugna tendía a ser abierta o larvada impidiendo una colaboración constructiva por culpa de la visibilidad o los problemas derivados de la cercanía o lejanía con el gobierno de turno, lo que impedía hacer más eficientes los recursos o adoptar posiciones comunes europeas en temas complejos como los DD.HH y la lucha contra la corrupción o el narcotráfico.

Aunque mi experiencia personal es mayoritariamente latinoamericana y soy consciente de sus particularidades, no trasladables necesariamente a los países ACP o a Asia, sí creo reflejar algunos de los problemas estructurales que nos han impedido trabajar más armónicamente sobre el terreno. Si bien es cierto que la PESC y la política exterior de la Unión han estado basadas en el principio de la unanimidad, con mayorías cualificadas en algunos casos, frente al método comunitario del primer pilar, para los que sentimos a Europa como una fuerza motora y trasformadora sin vuelta atrás, nuestro espíritu federalista nos empujó siempre a hablar desde España como parte comprometida con Europa y con el claro objetivo de hacer más fuerte y pro-

${ }^{10}$ Para un primer diagnóstico en esa dirección: LAATIKAINEN, K. y K.E. SMITH (eds.), Intersecting Multilateralisms: The European Union at the United Nations, Palgrave, Basingstoke, 2006.

${ }_{11}$ Para un interesante análisis vid.: EMERSON, M. y WOUTERS, J., «The EU's Diplomatic Debacle at the UN: What else and what next?», CEPS Commentary, 2010, disponible en: http://www.ceps.eu

12 Vid. p.ej. NOGUEIRA BATISTA, P., «Europe Must Make Way for a Modern IMF» en Financial Times, 24 de septiembre de 2010, p. 9 
minente el perfil europeo. Pero dejar todo a la buena voluntad, las habilidades o simpatías de los embajadores era y será siempre un riesgo muy alto.

Como recuerda Paola Bilancia, de acuerdo con los tratados en vigor, los Estados deben «apoyar activamente y sin reservas la política exterior y de seguridad común con lealtad y recíproca solidaridad. Deben, además, conformar sus respectivas políticas nacionales a lo establecido en las posiciones comunes y en las acciones comunes, y pueden someter al Consejo las cuestiones concernientes a la PESC». La misma autora argumenta que parte del éxito de esta ecuación va a depender de la sensibilidad y habilidad de la Alta Representante que deberá conservar la confianza del Consejo Europeo, negociar dentro de la Comisión y dar la cara ante el Parlamento evitando al máximo las fricciones y gestionando las diferencias de manera constructiva ${ }^{13}$.

El nuevo SEAE nace sin duda con mayor complejidad, pero con mucho mayor nivel de definición y a base de integrar la mejor experiencia de gestión y acción de todos los miembros y las instituciones comunitarias. La opción es jugar a fortalecer ese esquema, a liderarlo y a influenciarlo o, por el contrario, a ponerle trabas, debilitarlo o hacerlo inocuo ${ }^{14}$. Creo sinceramente que el reto de una potencia media influyente como España, debe jugar la primera baza aunque sintamos temporalmente la pérdida de protagonismo en algunas áreas geográficas pero a cambio de ganarlo en muchas otras, permitiéndonos hacer más eficiente y racional nuestro despliegue internacional y la defensa de nuestros intereses que están representados directamente en más de cien países pero que tiene implicaciones universales.

Un esfuerzo de esta naturaleza requiere de un abordaje sistemático de los retos relacionados con el funcionamiento, despliegue y maduración del SEAE sobre el terreno y de nuestro propio Servicio Exterior al interior de la administración española, aspectos sobre los que se pueden hacer algunas consideraciones.

\section{Los retos frente al despliegue exterior de la UE}

- La representación múltiple que asumen desde enero de este año los Embajadores de la UE, clarifica enormemente el trabajo y debe de reducir tensiones funcionales y protocolares. De entrada, los Emba-

13 Ver BILANCIA, P., «Posibles desarrollos de la política exterior y seguridad común tras el Tratado de Lisboa» en ReDCE, n. ${ }^{\circ}$ 13, Enero-Junio 2010.

${ }_{14}$ Un análisis sugerente sobre esos y otros escenarios abiertos tras la creación del SEAE en: BATORA, J., «A Democratically Accountable European External Action Service: Three Scenarios» en European Integration online Papers, vol. 14, 2010. Disponible en: http://eiop. or.at/eiop/index.php/eiop/article/view/2010_013a 
jadores europeos son seleccionados de manera transparente y competitiva, con criterios de calidad y especialidad y sus tareas y responsabilidades aparecen mucho mejor definidas. Su rotación frecuente, la categorización y especialización de los funcionarios y secciones en las nuevas Embajadas crean un sistema totalmente compatible con el de los países miembros. Probablemente, el tamaño de las Embajadas europeas será muy semejante en cada país al de las representaciones de los miembros más importantes, lo que permitirá poder interactuar de igual a igual y, además, tendrán dotaciones capaces de auxiliar y representar a aquellos países cuyas presencias sean mínimas, simbólicas o trabajen en acreditación múltiple.

- A la vista de tan importante despliegue, cada país debería revisar su aparato exterior político, comercial, cultural y consular, por señalar solo algunas áreas de presencia regular y más tradicional. Sería deseable que en cada país y circunstancia cada uno de los miembros vaya decidiendo cómo quiere estar frente a la potente maquinaria de la UE. Es probable que se generalicen para los países menores las acreditaciones múltiples, o las puras presencias sectoriales (Consulados, Centros Culturales u oficinas económicas) o las «Embajadas albergue» con presencia de funcionarios de varios países en función de las perspectivas bilaterales sin tener que pensar en costosos despliegues. Para los países de nivel medio o grande la oportunidad es única para declinar presencias en lugares de escaso interés bilateral o de complejidad política y cultural en los que las posiciones comunes en temas delicados, como por ejemplo los derechos humanos o la lucha contra la corrupción, podrán ser vehiculadas de manera más efectiva por una sola voz. En resumen, este formidable despliegue europeo debería repercutir en un examen profundo de los mapas de presencia de cada miembro en el mundo.

- Cada país miembro debería tener interés en colocar o posicionar en el SEAE y en países concretos y afines a sus intereses, a cuadros cualificados que valoricen culturas y presencias concretas y hagan más afinada la acción y el discurso europeo. En el caso español, es obvio que esto se tendrá que dar en las Embajadas europeas en América Latina y el Mediterráneo donde frecuentemente somos los primeros donantes e inversores externos. Tengo la impresión inicial que el conocimiento y la experiencia de países, idiomas o problemáticas están siendo tomadas en cuenta en este primer proceso de selección sabiendo que del prestigio y desempeño de estas primeras embajadas europeas en el mundo va a depender el nivel de respetabilidad en los países receptores y ante los veintisiete. 
- Aunque las competencias e instrumentos de la AR y el SEAE están claramente definidos: cooperación al desarrollo, democracia y DD.HH, vecindad y asociación, cooperación con países industrializados, seguridad nuclear y estabilidad, el proceso de decisión y gestión tendrá que recorrer un largo recorrido (campo-sede-campo) que demandará afinar los procedimientos de trabajo. Las dinámicas de comunicación y coordinación deberán ser revisadas y mejoradas. Lo mismo tendrá que hacerse entre las Representaciones en Bruselas y las Embajadas bilaterales para una buena toma de decisiones. A las tradicionales reuniones de Jefes de Misión, Consejeros y grupos especializados, deberán sumarse las de especialistas, los grupos focales con actores locales de la sociedad civil, los estudios y encuestas compartidas y otros instrumentos novedosos de coordinación y apropiación de las realidades locales. Las presidencias rotatorias quedan prácticamente obsoletas en los temas de competencias de la AR, aunque se mantienen en el resto y la interlocución con los gobiernos, que en sus presidencias ejercitaban los miembros, o las llamadas troikas deberán adaptarse a nuevos y flexibles formatos.

- Temas de gran relevancia serán los relativos a la gestión de los recursos de cooperación que deberían ser mucho más coordinados y focalizados en las áreas prioritarias como la promoción de la institucionalidad democrática, los fondos de emergencia y las políticas relacionadas con el cumplimiento de los ODM, la reducción de la deuda y los DD.HH, entre otras. Más compleja será la articulación en aquellos temas que no son de la estricta competencia de la AR, pero que definen la imagen corporativa de la UE en muchos países y ante el sistema multilateral como los de inversión extranjera, medio-ambiente, I+D, género, promoción de la cultura etc. que han ido ganando mucho espacio en la agenda aunque hay que reconocer que en muchos de ellos se han ido dando pasos hacia una cierta convergencia. Y mucho más difícil por fin, será la coordinación en los temas de inteligencia, seguridad, lucha contra el narcotráfico y cooperación militar que tienen claves propias y de alta sensibilidad pero que admiten márgenes importantes de acercamiento aunque está prevista en el Tratado de Lisboa una cooperación reforzada en materia de defensa.

- Partiendo del momento especial que vive el proyecto europeo y de la complejidad del nuevo despliegue, habrá que darse un plazo amplio, mínimo entre tres y cinco años, para empezar a ver resultados avanzados, digerir los cambios y detectar los nichos de problemas tradicionales o emergentes. En todo caso, hay que hacer una apelación a la buena voluntad de todas las partes, y al hecho incontestable que el Tratado de Lisboa es nuestra plataforma constitucional co- 
mún vigente. Por otra parte, la capacidad, el compromiso y el talento de los cuadros europeos hay que darla por descontada. Habrá países, circunstancias y funcionarios más o menos proclives hacia el nuevo SEAE, pero hay que apostar por su éxito y viabilidad, pensando que muy bien podría ocurrir que ante los problemas de otorgar por el momento un alto perfil a la AR, este déficit pueda ser compensado o puesto en valor por la actitud y el desempeño de cada embajada europea en particular y de la maquinaria en su conjunto. La ciudadanía europea tiene que empezar a observar las ventajas del SEAE en sus viajes, sus inversiones, sus intercambios educativos, en el posicionamiento ante las emergencias y los retos mundiales, en sus problemas consulares y en la difusión de nuestros valores europeos lo que se consigue no solo con discursos y tomas de posición institucionales sino también con presencias eficientes sobre el terreno.

\section{Los retos al interior de los servicios exteriores}

En mi opinión, y coincido con partes sustanciales del estudio de Clara Portela respecto a las implicaciones del SEAE para España ${ }^{15}$, cada servicio exterior y diplomático individual europeo tiene ante sí la oportunidad clara, e incluso la obligación, para reformularse a la luz de este nuevo reto ${ }^{16}$. Algunas de las orientaciones o tendencias deberían venir de las siguientes acciones:

- A España y creo sinceramente a los demás países de la UE, les interesa un SEAE fuerte y representativo de la pluralidad y los valores europeos. Dicha definición empujaría a asegurar que nuestros mejores cuadros diplomáticos y especialistas deberían rotar, entrar y salir, en ambos sistemas para fortalecerlos mutuamente.

- Los estudios especializados y la preparación de cuadros deberán ser revisadas y adaptados a las nuevas circunstancias. A falta, lo que sería ideal, de una Escuela Europea del Servicio Exterior habrá que fortalecer y reorientar las nacionales incluyendo aquellos temas económicos, de desarrollo y cooperación, estratégicos y culturales que quedan impregnados por las nuevas circunstancias y el aprendizaje

15 Vid. PORTELA, C., «El Servicio Exterior de Acción Europeo: implicaciones para la reforma del servicio diplomático español», Documento de trabajo OPEX. Fundación Alternativas. 2009. Disponible en: www.falternativas.org/content/download/15434/.../3/.../SEE.pdf

16 Sobre la adaptación paulatina de los ministerios de asuntos exteriores de los Estados miembros a los imperativos de la integración Europa vid el trabajo pionero de: HOCKING, B. y SPENCE D. (eds.), Foreign Ministries in the European Union, Palgrave, London, 2002. 
de nuevas habilidades (negociación, liderazgo, idiomas exóticos, gerencia, nuevas tecnologías etc.) que busquen la excelencia, la adaptabilidad y la especialización incremental.

- Los propios sistemas diplomáticos nacionales deberían tener un interés genuino en abrirse y fortalecerse con diferentes fórmulas (contrataciones temporales, segundos turnos, préstamos y canjes profesionales etc.) a especialistas de diferentes ramas de saber con experiencias acumuladas en el sector académico, empresarial, cultural, cooperación y multilateral etc. Sería razonable, además, pensar en nuevos mecanismos de ingreso, formación y promoción de cuadros, revisando las categorías tradicionales para incorporar investigadores, analistas, comunicadores y gestores, entre otras formaciones, de muy diversa procedencia. Volveríamos a la idea, que he defendido siempre, que se debería tender más a un servicio exterior en sentido amplio, con diferentes ventanas y opciones profesionales, que al sostenimiento de un servicio diplomático puro y tradicional. Este es un tema aún pendiente en la agenda de reformas del Ministerio de Asuntos Exteriores.

- Particularmente la ejecución de los programas culturales y de cooperación deberá ser reconsiderada. En función de las prioridades programáticas y sectoriales que tengamos en cada país, convendría declinar el liderazgo y la coordinación a las embajadas de la UE y, en todo caso, unificar y coordinar criterios de priorización, programación general y sectorial, evaluación y auditoria de acuerdo con los criterios surgidos del CAD. La presencia de centros culturales, que en algunos casos podrían compartirse, colegios nacionales, sistemas de becas e intercambios etc..., deberían ir sufriendo modificaciones y adaptaciones para superar la cacofonía actual. Al igual que empieza a ocurrir en otros Estados miembros, nuestro particular sistema autonómico, con sus diferentes expresiones exteriores, debería ser tenido en cuenta en este rediseño ya que es un fenómeno instalado y de creciente fortaleza en los ámbitos cultural, comercial y de promoción de las colectividades de ámbito regional ${ }^{17}$.

- Por fin, quedaría el espinoso tema de la compatibilidad de la visibilidad europea y la singular de cada país que por mucho tiempo tendrán que buscar formulas de complementariedad y articulación en vez de competir abiertamente. Convendría a este respecto ver como, con gran sutilidad y dificultad, se ha ido abordando este tema en el

17 Vid. CORNAGO, N., «On the normalization od Sub-State Diplomacy» en The Hague Journal of Diplomacy, vol. 5, 2010, pp. 11-36. 
complejo mundo de las Agencias y Fondos de las NN.UU (One UN, delivering as one etc.) o en programas entre donantes del Norte y el Sur y agencias multilaterales (sistema multibilateral o cooperación triangular) para sacar algunas conclusiones y lecciones aprendidas ${ }^{18}$.

\section{Conclusión}

El SEAE es una apuesta mayor y sin vuelta atrás y que a pesar de surgir en este momento turbulento no debe ser obstruida o desvalorizada. Bien al contrario, es algo que muchos hemos estado esperando y deseando desde la necesidad de dar racionalidad, coherencia y visibilidad a la UE y desde la exigencia de influir de manera más activa y eficaz en la realidad mundial. Nuestra política exterior, acompañada hoy de un fuerte componente empresarial, de cultura y cooperación y que, además, tiene la fortaleza de un idioma en expansión, no debería tener ningún temor a esta oportunidad que, de manera natural, podría tener algunas improntas españolas muy valiosas en el terreno de la solidaridad, el diálogo de civilizaciones y la cultura de paz, la modernización democrática, la igualdad de género y los DD.HH. Y también en otras más lúdicas derivadas de nuestro liderazgo por ejemplo en el turismo, la gastronomía, el cine y las actividades artísticas y deportivas. Ojalá esa Europa «capaz de hablar con una sola voz y ejercer su peso en las grandes cuestiones mundiales, pueda, al fin, realizarse, tras largas alternancias de esperanza y decepción», como hace más de veinte años soñaba Enrique Barón, entonces Presidente del Parlamento Europeo, en su intervención en 1990 ante el Consejo Europeo de Roma ${ }^{19}$.

18 Un reciente trabajo de singular interés sobre esta cuestión nos lo ofrece: EMERSON, M. y KACYINSKI, P.M., Looking afresh at the external representation of the EU in the international arena, post-Lisbon, CEPS Policy Brief, No 212, Brussels, July 2010.

19 Vid. BARÓN, E., Europa. Pasión y razón, Biblioteca Nueva, Madrid, 2005, p. 76. 